\section{Vaccinia's interactions}

A comprehensive two-hybrid analysis has identified 28 previously unknown interactions among the proteins encoded by the vaccinia virus genome (Proc. Natl. Acad. Sci. USA, 97, 4879-4884, 2000). Vaccinia has been a useful tool in medicine and biotechnology, from its early use as the smallpox vaccine to its current uses to manufacture recombinant proteins and as a vector for recombinant vaccines against cancer and infectious disease. Using the array-based format developed by Stan Fields's laboratory at the University of Washington in Seattle (Nature, 403, 623-627, 2000), Steven McCraith and coworkers have systematically tested each of the 70,000 possible pairwise combinations of the virus's 266 putative protein-coding genes for the ability to interact in the two-hybrid assay. Although confirmation of the validity of these new interactions will be necessary, several new leads have been uncovered in the quest to gain a better understanding of vaccinia replication. For a subset of proteins, however, neither sequence homology searches nor two-hybrid analysis has revealed any information as to their function. "Developing the next generation of technologies to approach this type of problem will be one of the future challenges in the arena of genomic analysis," states McCraith.

$R F$

\section{Cold fusion for eggs}

Novel methods of membrane fusion may help women who have fertility problems due to eggs that contain defective cytoplasm. The techniques, a new method of mechanically induced membrane fusion in human oocytes and a previously described chemical fusion method, were developed by a collaboration of researchers from France, Spain, and Italy and essentially involve transferring the nucleus of a defective egg into a donor egg (Hum. Reprod., 15, 1149-1154, 2000). Most cell fusion techniques used in mammalian cloning employ electrofusion, which cannot be used for human oocytes because it activates them. In the current study, the team used a modified chemical polyethylene glycol method to essentially "glue" together two zona pellucida-free human eggs, one of which was enucleated. It also used a mechanical method performed by making a hole in the zona and then manipulating the eggs together using a micropipette.

Research News Briefs written by Alan Dove, Robert Frederickson, Julie Grisham, and Andrew Marshall.

\title{
Soluble receptor against lupus?
}

A soluble form of a receptor for a cytokine that activates B cells is effective for inhibiting the development of autoimmune disease in a mouse model of systemic lupus erythematosus (SLE), according to a study recently published in Nature (404, 995-999, 2000). The results validate a genomic screening approach for identifying protein ligands and may open a promising avenue to treating human autoimmune diseases. Researchers at ZymoGenetics (Seattle, WA) first showed that the cytokine (zTNF4), a member of the tumor necrosis factor (TNF) ligand family, causes SLE-like symptoms in mice when overexpressed. They then identified TACI, a receptor for zTNF4, by screening a cDNA expression library prepared from B cells with a labeled form of the cytokine. Reasoning that the TACI-zTNF4 interaction could be of potential therapeutic value, the researchers then set about creating a soluble form of TACI and tested it in the mouse SLE model. The treatment inhibited the development of proteinuria in the animals and prolonged

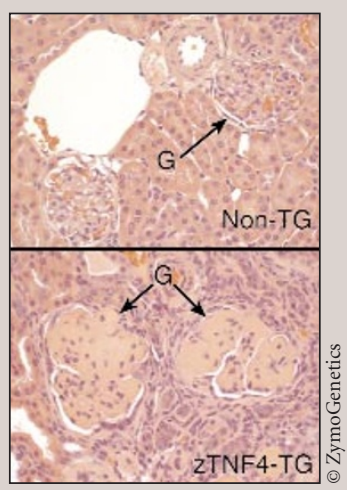

Shown: Kidney sections; $\mathrm{G}=$ glomerul their survival. Although the results are promising, the researchers acknowledge that more work must be done to determine the possible side effects of inhibiting B-cell activation with TACI. "We are currently testing the effects of TACI treatment on the development of a normal immune system and during a primary and secondary immune response," says Jane Gross, a company scientist and lead author on the paper.

The authors say the intracytoplasmic sperm injection (ICSI) technique "will probably be the optimal way of fertilizing the reconstructed oocytes." The technique also has potential for use in therapeutic cloning.

\section{Surrogate $\beta$-cells}

New work indicates that the liver can be induced to serve as a surrogate for the pancreas, with a little help from the PDX-1 gene. By injecting mice with an adenoviral vector containing the gene encoding PDX-1, a homeodomain protein involved in the development and differentiation of pancreatic cells, a group of researchers from Israel has succeeded in converting a subpopulation of liver cells to a pancreatic $\beta$-cell phenotype. Following adenoviral treatment, about $60 \%$ of the mice liver cells started synthesizing PDX-1, with a 25fold increase in insulin expression, 59\% of which was fully processed functional protein. In an experimental model of diabetes in which hyperglycemia was induced by the chemical streptozotocin, the researchers found that only those mice treated with the PDX-1 gene were able to survive and ameliorate glucose dysregulation. It is not yet clear whether the surrogate $\beta$-cells can respond normally to fluctuations in glucose or whether, like $\beta$-cells, they themselves will become targeted by autoimmune processes. But according to Axel Kahn, a diabetes researcher at INSERM (Paris), the results "could constitute a breakthrough in the prospects of therapy for type I diabetes," which currently affects up to 14 million people worldwide. The findings are reported in Nat. Med. (6, 568-572, 2000).

$A M$

\section{High-affinity TCRs}

Using an in vitro evolution technique, researchers at the University of Chicago (Chicago, IL) and the University of Illinois (Urbana, IL) have developed T-cell receptor (TCR) proteins with an extremely high affinity for a target peptide-MHC ligand. Besides demonstrating that there is no intrinsic structural block to generating high-affinity TCRs, the findings could have implications for a variety of clinical applications. The approach, described in Proc. Natl. Acad. Sci. USA (97, 5387-5392, 2000), relies on the surface expression and display of mutant single-chain TCRs in a library of yeast cells. By selecting TCRs that bound to a model nine-residue antigen (QL9) from this pool, the researchers were able to avoid the selective pressures that appear to limit TCR affinity in vivo. The team was able to isolate TCRs with an affinity for QL9-MHC 100-fold higher than naturally occurring TCRs. A soluble form of the highaffinity TCR can detect peptide-MHC complexes on antigen-presenting cells, suggesting that the artificially generated TCRs could target these complexes therapeutically. "I see no reason why one couldn't perform immunohistology with these reagents in the same way one does with monoclonal antibodies," says David Kranz, senior author on the paper. $A D$ 